\title{
The role of immunohistochemical examination in diagnosis of papillary thyroid cancer in struma ovarii
}

\author{
Ewelina Szczepanek-Parulska ${ }^{1}$, Anna Pioch ${ }^{1}$, Ewa Cyranska-Chyrek, ${ }^{1}$, \\ Kosma Wolinski', Donata Jarmolowska-Jurczyszyn' ${ }^{2}$, Malgorzata Janicka-Jedynska ${ }^{2}$, \\ Przemyslaw Majewski², Maciej Zabel ${ }^{3,4,5}$, Marek Ruchala ${ }^{1}$
}

${ }^{1}$ Department of Endocrinology, Metabolism and Internal Medicine Poznan University of Medical Sciences, Poland

${ }^{2}$ Department of Clinical Pathology, Poznan University of Medical Sciences, Poland

${ }^{3}$ Department of Anatomy and Histology, University of Zielona Gora, Poland

${ }^{4}$ Department of Histology and Embryology, Wroclaw Medical University, Poland

${ }^{5}$ Department of Human Morphology and Embryology, Wroclaw Medical University, Poland

\begin{abstract}
Introduction. Struma ovarii (SO) is a monodermal teratoma in which thyroid tissue comprises more than 50\% of the tumour. Papillary thyroid cancer (PTC) in SO is a rare finding, as only 5\% of SO cases undergo malignant transformation. Malignant SO is usually asymptomatic and infrequently diagnosed preoperatively. Because of its rarity, there is no consensus about diagnosis and management, while treatment and follow-up procedures are not clearly established.

Material and methods. Herewith, we report two cases of PTC in SO. The first patient was a 25-year-old woman diagnosed with bilateral ovarian tumours. The second patient, 19-year-old woman, presented with unilateral ovarian mass. Both patients were qualified for surgical excision of the tumours. Histopathological specimens underwent both conventional histopathological assessment and immunohistochemical staining.

Results. In the first patient histopathology revealed SO with two foci of PTC. Immunohistochemically a positive expression of CK7, CK19, p63 and thyroglobulin (Tg) confirmed the diagnosis. She underwent total thyroidectomy in 2016 in order to enable ablative radioiodine therapy and facilitate further thyroglobulin monitoring. Unfortunately, the patient was lost from follow-up. In the second patient, histopathological diagnosis was follicular variant of PTC in SO. Postoperatively, a pelvic CT revealed osteolytic lesion $6 \mathrm{~cm}$ in size, being a metastatic change. The patient underwent unilateral ovariectomy, total thyroidectomy and multiple cycles of radioiodine therapy. Currently, 9 years following the diagnosis, the patient achieved disease remission.

Conclusions. PTC in SO still remains a diagnostic and therapeutic challenge. Immunostaining for CK7, CK19, p63 and Tg might be helpful in histopathological diagnosis. The decision on the need of total thyroidectomy and radioiodine therapy should be made individually. However, thyroid remnant ablation increases the sensitivity and specificity of follow-up testing using serum Tg level as a tumour marker. (Folia Histochemica et Cytobiologica 2019, Vol. 57, No. 1, 35-41)
\end{abstract}

Key words: struma ovarii; papillary thyroid cancer; teratoma; CK7; CK19; p63; thyroglobulin; IHC

Correspondence address:

Prof. Ewelina Szczepanek-Parulska, MD, PhD

Department of Endocrinology, Metabolism and Internal Medicine

Poznan University of Medical Sciences

Przybyszewskiego 49, 60-355 Poznan, Poland

tel. + 486186913 30; fax: +48 618691682

e-mail: ewelinaparulska@gmail.com

\section{Introduction}

Germ cell tumours are a heterogeneous group of neoplasms arising from primordial germ cells. The most common type of germ cell tumours are teratomas [1]. Struma ovarii (SO) is a monodermal germ cell tumour characterized by the presence of thyroid 
tissue in more than 50\% [2]. It was first described in 1899 by Boettlin, who observed the presence of thyroid follicular tissue in the ovaries [3]. It is most often diagnosed postoperatively during histopathological examination, while radiological imaging is unspecific [4]. Strumae ovarii are one of the rarest ovarian tumours - they represent $1 \%$ of them and comprise $2-5 \%$ of teratomas [5]. Less than 200 cases have been so far reported in medical literature [2]. Struma ovarii is usually a benign tumour, being histologically and pathologically identical to normal thyroid tissue found in typical localization. Less than $5 \%$ of these tumours undergo malignant transformation [2,6-8]. The age at presentation of SO is usually between 40 and 60 years $[3,9,10]$. The most common type of cancer within SO is well-differentiated thyroid cancer, most commonly, papillary thyroid cancer (PTC) $[9,11]$. The diagnosis of thyroid cancer arising in SO should be based on the similar criteria as for primary thyroid gland disease.

Due to the rarity of this type of tumour, there has been a paucity of data in the medical literature about its optimal diagnosis, treatment and follow-up. Classically, the treatment is the surgical resection of the ovarian mass. Iodine-131 therapy can be proposed after total thyroidectomy, but no consensus exists in terms of surveillance and adjuvant treatment [12]. Diagnostic difficulties may appear also at the level of histopathological examination. In this report we present two patients diagnosed postsurgically with thyroid cancer in SO and demonstrate the pivotal role of immunohistochemical (IHC) staining for final diagnosis.

\section{Material and methods}

Herewith we report two cases of PTC in SO. The first patient was a 25-year-old woman diagnosed with bilateral ovarian tumours. She was suffering from recurrent acute lower abdominal pain. Similar episodes of pain were experienced during sexual intercourses in the previous two years, since she gave birth to a first child via caesarean section. There were no other associated gastrointestinal or genitourinary symptoms. She was otherwise healthy with nonsignificant past medical history. The second patient, 19-year-old woman, presented with unilateral ovarian mass. Both patients underwent clinical assessment, imaging studies and were qualified for surgical excision of the ovarian tumours. Histopathological specimens underwent both conventional histopathological assessment and IHC staining.

Immunohistochemistry. Formalin fixed, paraffin embedded tissue specimens were cut into $4 \mu \mathrm{m}$ sections. The sections were mounted on adhesion microscope slides (Dako, Glostrup, Denmark). Deparaffinization, rehydra- tion and antigen retrieval was achieved by incubation in a water bath (PT Link, Dako) in a buffer (High pH, EnVision FLEX Target Retrieval Solution, Dako) at $97^{\circ} \mathrm{C}$ for $20 \mathrm{~min}$. The sections were then machine stained with the use of Autostaner Link 48 using a visualization kit for IHC staining (EnVision FLEX+, Mouse, Dako). Prediluted primary antibodies included: mouse monoclonal anti-Human Cytokeratin 7 (clone: OV-TL 12/30), monoclonal mouse anti-human cytokeratin 19 (clone RCK108, Dako), monoclonal mouse anti-human p63 protein (clone: DAK-p63), polyclonal rabbit anti-human thyroglobulin (Dako), and Rabbit Linker (Dako) for amplification. Then, the slides were dehydrated and mounted with cover glass with Mounting Medium (Dako).

\section{Results}

In the first patient, an ultrasound examination revealed normal sized and shaped uterus and endometrial thickness of $4 \mathrm{~mm}$. Moreover, left ovarian cyst $4.7 \mathrm{~cm}$ in size with dense content and $8.7 \mathrm{~cm}$ mass behind the uterus suggestive of a teratoma of a right ovary, were revealed. Ultrasound pattern was compatible with presumably benign tumours; hence, there was no need to conduct computed tomography (CT) scans or magnetic resonance imaging (MRI). The patient was qualified for surgery; due to the size and bilateral location of the lesions, the patient was qualified for conventional laparotomy. During surgical procedure, abdominal cavity and uterus were normal, while the ovaries were enlarged and presented bilateral masses which were enucleated and removed. Intraoperative histopathological examination revealed a teratoma on the right side $(9.0 \times 6.0 \times 5.0 \mathrm{~cm})$ with $\mathrm{SO}$ and dermoid cyst in the left ovary $(8.0 \times 7.8 \times 2.0 \mathrm{~cm})$. In the right ovary mass the biggest cyst was $3 \mathrm{~cm}$ in size and was filled with clear serous fluid. In the other cysts there was yellow, viscous material. Patient's postoperative period was uneventful.

Microscopic examination of the surgical specimen prepared by haematoxylin and eosin (H\&E) staining (Figs. 1A, B) revealed two foci of PTC in SO (the largest $4 \mathrm{~mm}$ in size). The diagnosis was confirmed by an IHC examination. Immunohistochemically, tumour cells were strongly positive for thyroglobulin (Tg), cytokeratin 19 (CK19), cytokeratin 7 (CK7) and p63 (Figs. 2A-D). Based on all these findings, the diagnosis was papillary thyroid cancer arising within SO. Subsequently, in order to enable potential ablative radioiodine therapy and facilitate further $\mathrm{Tg}$ monitoring, the patient was referred for total thyroidectomy. The patient had two mixed solid/cystic focal lesions in the thyroid (size $8 \mathrm{~mm}$ and $11 \mathrm{~mm}$ ) detected on thyroid ultrasound examination. Both presented 

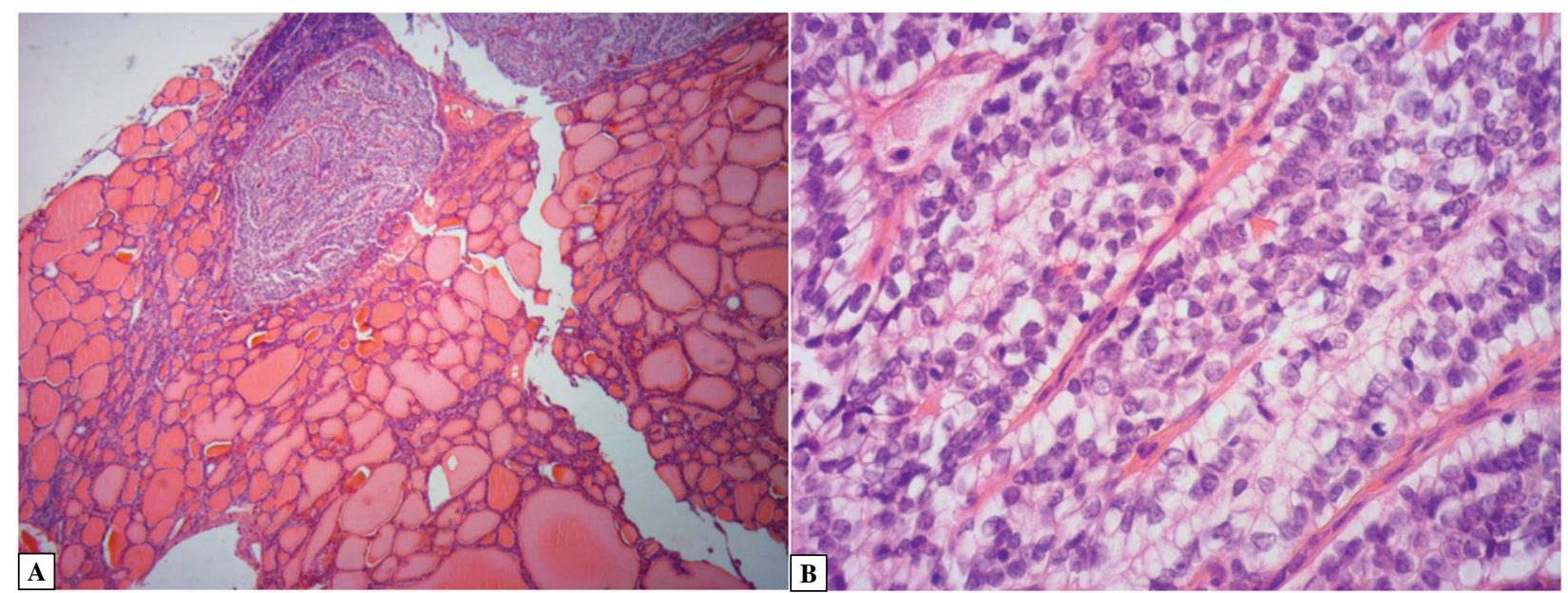

Figure 1. Microscopic view of papillary thyroid cancer in struma ovarii of the Patient 1 . H\&E staining. Total magnification A. $40 \times$ and B. $400 \times$.
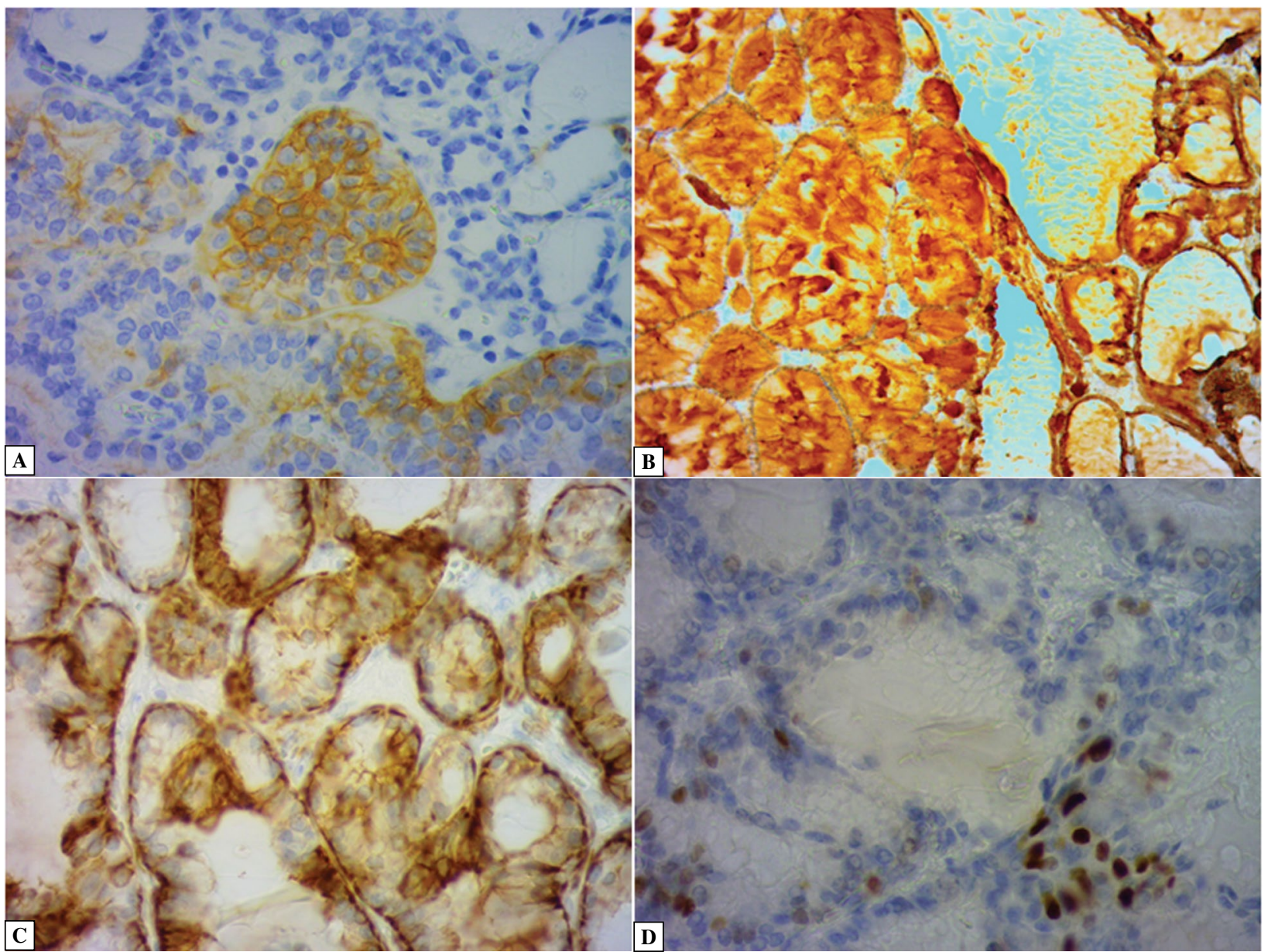

Figure 2A. Immunohistochemical (IHC) detection of the studied antigens in the tissue of papillary thyroid cancer in struma ovarii of the Patient 1. A. CK19 positive immunoreactivity in the tumor tissue, while negative in the surrounding non-cancerous thyroid tissue. B. Immunoreactivity of thyroglobulin. C. Immunoreactivity of cytokeratin 7. D. Immunoreactivity of p63. Immunohistoch-emical stainings were performed as described in Methods. Total magnification $400 \times$. 
no sonographic features of malignancy and were diagnosed as benign colloid nodules on cytological examination of the specimen obtained on fine-needle aspiration biopsy. On postsurgical histopathological examination there were no malignant lesions found in the thyroid, and colloid goitre was diagnosed. After surgery the patient was treated with oral L-thyroxine therapy and the post-surgical serum $\mathrm{Tg}$ concentration on suppressive doses measured 6 week following surgery was $0.9 \mathrm{ng} / \mathrm{ml}$, marking the low risk of persistent disease. The patient was scheduled for whole-body scintiscan and stimulated Tg assessment 3-6 months following surgery in order to decide whether to administer radioiodine therapy. Unfortunately, the patient was lost from follow-up. Still, in this patient a long-term regular follow up is required in order to monitor $\mathrm{Tg}$ concentration and including regular gynaecological visits.

The second patient had an unilateral ovarian mixed solid and cystic mass $(4.0 \times 3.0 \times 6.8 \mathrm{~cm})$ diagnosed during routine gynaecological follow-up. An excision of the lesion was performed from laparoscopic approach. Histopathological diagnosis was teratoma of the size $6 \times 7 \times 4 \mathrm{~cm}$ that contained focus of follicular variant of PTC in SO (size not reported, histopathological material unavailable). Two months after the surgery, laparoscopic complete right ovariectomy was performed and two months later total thyroidectomy was performed. No focal lesions were detected in the thyroid preoperatively on ultrasound examination or postoperatively on histopathological examination. Radioiodine therapy was then introduced $-200 \mathrm{mCi} 131-\mathrm{I}$ was administered. Few months later pelvic CT scan revealed an osteolytic lesion in the left ilium measuring $4.8 \times 5.8 \times 1.0 \mathrm{~cm}$, being a metastatic change. Whole-body scintiscan depicted radioiodine uptake in the metastasis. During 9 years of follow-up the patient was on constant L-thyroxine suppressive therapy and received cumulative activity at the level of $1400 \mathrm{mCi}$ of radioiodine. At the moment she achieved complete biochemical and structural disease remission.

\section{Discussion and conclusions}

Most patients with SO present no clinical symptoms and are diagnosed incidentally. In some patients, as it was in the two presented cases, they may cause symptoms similar to those typical of other ovarian tumours, such as low abdominal pain, nausea, vaginal bleeding and abnormal menstrual cycles $[5,6]$. Sometimes, the first symptom is palpable abdominal mass $[5,7,13]$. The late manifestation of malignant $\mathrm{SO}$ are signs of metastatic disease such as back pain
$[14,15]$. Dissimilar from previous reports, in two of our patients SO was diagnosed in their $19^{\text {th }}$ and $25^{\text {th }}$ years of age (in comparison to average 40-60 years reported in the literature). The most common type of cancer detected in this type of teratoma is PTC, as it was in our patients. In one of them follicular variant of PTC was diagnosed at the stage of distant bone metastasis. Distant metastases from malignant SO are highly uncommon and occur in only about $5 \%$ of cases [16]. Tumours can metastasize via direct spread to the omentum, peritoneal cavity and contralateral ovary, via the lymphatic system to pelvic and para-aortic lymph nodes and via blood to the bones, brain, lung, contralateral ovary and liver [16]. Bone is a very rare site for metastasis and there are only a few cases in the English language literature which have reported metastasis to the bones [17-19]. The metastatic pattern of malignant SO resembles that of ovarian cancer; therefore, for advanced disease certain authors propose performing complete staging surgery as for ovarian cancer.

Similarly to both our patients, over $92 \%$ of the patients with thyroid cancer in SO are clinically and biochemically euthyroid [3, 20, 21] However, $83 \%$ of patients who present with hyperthyroidism already had distant metastases from malignant $\mathrm{SO}$ [22]. There is no typical ultrasound feature of SO, although it could be suspected if there is a large solid mass on ultrasound examination, as it was in both our patients. Tumour markers are not useful and they represent low clinical value in these cases. The symptoms are also non-specific; therefore, most cases of malignant SO have subclinical course and are diagnosed incidentally. Most frequently the patients present with pelvic pain. Postsurgical assessment of thyroglobulin can be used for follow-up as a marker of response to treatment, especially in cases of metastases.

Both papillary and follicular thyroid cancers arising in SO have been reported in medical literature [16]. The most common type of malignant SO is PTC $[15,23,24]$. The pathological diagnosis of malignant SO is primarily established according to the criteria for tumours of the thyroid gland. These criteria include ground glass overlapping nuclei and nuclear grooves, or mitotic activity and vascular invasion $[8,15]$. The diagnosis of thyroid cancer in SO might be challenging. The "gold standard" in diagnosis of thyroid cancers is using routine $\mathrm{H} \& \mathrm{E}$ staining. Immunohistochemistry is an additional procedure, often necessary to provide diagnosis. Besides H\&E staining for evaluating thyroid tumour morphology, immunohistochemical methods have been widely used [25]. Immunohistochemical markers are of two types: those related to proteins present in normal 
thyrocytes (such as thyroglobulin and thyroid transcription factor-1) and those related to proteins in pathological thyrocytes (such as CK 19 and Hector Battifora mesothelial epitope-1) [26, 27].

Cytokeratins (CK), mainly expressed in epithelial cells and skin appendages, constitute the largest subgroup of intermediate filament proteins [28]. They participate in the formation of the cell skeleton and play an important role in the responses to stress, mitosis, the postmitotic period, cell signalling and movement, as well as in differentiation and apoptosis $[26,29]$. CK19 is mainly expressed in ductal epithelium (bile and pancreatic ducts, renal collecting ducts) and gastrointestinal epithelium [30]. Expression of CK19 might also be positive in pancreatic neuroendocrine tumours, hepatocellular cancer, cervical squamous cell cancer, thymic and lung cancers [31-34]. Malignant thyroid lesions present higher CK19 expression when compared to nonmalignant thyroid lesions [27]. In our patient, we confirmed that CK19 expression in the patient's tumour was positive suggestive of malignant process. Another marker demonstrating positive staining in our specimen was CK7. In the study by Bejarano et al., $79 \%$ of benign and malignant thyroid tumours were found to demonstrate positive reaction for CK7, especially all eight analysed PTCs [35].

There are only a few studies on the involvement of $\mathrm{p} 63$ protein in processes associated with thyroid tumour formation and they represent contradictory results. This protein is a member of p53 family of transcription factors having a major role in regulating proliferation and differentiation processes of epithelial cells [36]. Immunohistochemical expression of p63 is evident in some types of cancers including squamous cell cancer, choriocarcinoma and thyroid cancer [37-39], and was also demonstrated in our patient with PTC.

The optimal treatment guidelines for PTC arising in malignant $\mathrm{SO}$ are still disputable. Most authors advocate for aggressive treatment based on total abdominal hysterectomy with bilateral salpingo-oophorectomy, omentectomy and lymph node dissection [2, $6,8,24]$. Because of permanent infertility associated with this procedure, unilateral salpingo-oophorectomy/unilateral oophorectomy in order to preserve the patients' fertility is acceptable [2, 40]. As one of our patients was nulliparous and the second patient had one child, in both gynaecologic intervention was limited to an ovarian mass excision to preserve fertility and ovarian hormonal function. It is necessary to check the contralateral ovary in order to exclude any pathological changes. Moreover, the decision on completion thyroidectomy raises controversies. Debate remains about the role of total thyroidectomy and subsequent radioiodine ablation. Most authors suggest aggressive treatment with radioactive iodine therapy regardless of the presence or absence of metastases at time of diagnosis [2, 41, 42]. Some authors agree that thyroidectomy and radioactive iodine therapy should be undertaken only in case of recurrent disease or metastases [43]. In general, completion thyroidectomy and radioiodine ablation therapy appear to be well-accepted and effective treatments for malignant SO most often reported in the literature $[16,44]$, which also adheres to the standards applied to manage our patients.

Thyroid-type cancers arising in SO, especially the newly recognized entity, i.e. highly differentiated follicular cancer of ovarian origin, have a favourable prognosis with 5- and 25-year survival rate of $92 \%$ and $79 \%$, respectively $[8,43,45]$. Long-term follow-up with thyroglobulin and anti-thyroglobulin antibodies levels assessment is necessary due to the possibility of recurrence. Based on the rarity of these tumours and lack of firm prognostic factors, therapeutic decisions should be made individually, based on pathologic and clinical parameters, respecting the patient's informed consent.

In conclusion, PTC in SO still remains a diagnostic and therapeutic challenge. The diagnosis is most often made postoperatively. Immunostaining for CK7, CK19, p63 and thyroglobulin might be helpful in histopathological assessment. There is still no established treatment algorithm for SO patients. The decision on the need of total thyroidectomy and radioiodine therapy should be made individually. However, thyroid remnant ablation increases the sensitivity and specificity of follow-up testing using serum thyroglobulin levels as a tumour marker.

\section{References}

1. Sahin H, Abdullazade S, Sanci M. Mature cystic teratoma of the ovary: a cutting edge overview on imaging features. Insights Imaging. 2017; 8(2): 227-241, doi: 10.1007/s13244016-0539-9, indexed in Pubmed: 28105559.

2. Al Hassan MS, Saafan T, El Ansari W, et al. The largest reported papillary thyroid carcinoma arising in struma ovarii and metastasis to opposite ovary: case report and review of literature. Thyroid Res. 2018; 11: 10, doi: 10.1186/s13044-0180054-9, indexed in Pubmed: 30061934.

3. Yoo SC, Chang KH, Lyu MO, et al. Clinical characteristics of struma ovarii. J Gynecol Oncol. 2008; 19(2): 135-138, doi: 10.3802/jgo.2008.19.2.135, indexed in Pubmed: 19471561.

4. Lebreton C, Al Ghuzlan A, Floquet A, et al. [Thyroid carcinoma on struma ovarii: Diagnosis and treatment]. Bull Cancer. 2018; 105(3): 281-289, doi: 10.1016/j.bulcan.2017.11.014, indexed in Pubmed: 29459090.

5. Llueca A, Maazouzi Y, Herraiz JL, et al. Treatment and follow-up in an asymptomatic malignant struma ovarii: A case report. Int J Surg Case Rep. 2017; 40: 113-115, doi: 10.1016/j. ijscr.2017.09.005, indexed in Pubmed: 28982046. 
6. Lara C, Cuenca D, Salame L, et al. A Hormonally Active Malignant Struma Ovarii. Case Rep Oncol Med. 2016; 2016: 2643470, doi: 10.1155/2016/2643470, indexed in Pubmed: 27882257.

7. Oudoux A, Leblanc E, Beaujot J, et al. Treatment and follow-up of malignant struma ovarii: Regarding two cases. Gynecol Oncol Rep. 2016; 17: 56-59, doi: 10.1016/j. gore.2016.05.014, indexed in Pubmed: 27355004.

8. Zhu Yi, Wang C, Zhang GN, et al. Papillary thyroid cancer located in malignant struma ovarii with omentum metastasis: a case report and review of the literature. World J Surg Oncol. 2016; 14(1): 17, doi: 10.1186/s12957-016-0776-x, indexed in Pubmed: 26791568.

9. Goffredo P, Sawka AM, Pura J, et al. Malignant struma ovarii: a population-level analysis of a large series of 68 patients. Thyroid. 2015; 25(2): 211-215, doi: 10.1089/thy.2014.0328, indexed in Pubmed: 25375817.

10. Kraemer B, Grischke EM, Staebler A, et al. Laparoscopic excision of malignant struma ovarii and 1 year follow-up without further treatment. Fertil Steril. 2011; 95(6): 2124. e9-2124.12, doi: 10.1016/j.fertnstert.2010.12.047, indexed in Pubmed: 21269611.

11. Yassa L, Sadow P, Marqusee E. Malignant struma ovarii. Nat Clin Pract Endocrinol Metab. 2008; 4(8): 469-472, doi: 10.1038/ncpendmet0887, indexed in Pubmed: 18560398.

12. Luster M, Aktolun C, Amendoeira I, et al. European Perspective on 2015 American Thyroid Association Management Guidelines for Adult Patients with Thyroid Nodules and Differentiated Thyroid Cancer: Proceedings of an Interactive International Symposium. Thyroid. 2019; 29(1): 7-26, doi: 10.1089/thy.2017.0129, indexed in Pubmed: 30484394.

13. Qiao PF, Gao Y, Niu GM. Struma ovarii accompanied by mature cystic teratoma of the other ovary: A case report and literature review. Oncol Lett. 2015; 9(5): 2053-2055, doi: 10.3892/ol.2015.3029, indexed in Pubmed: 26137011.

14. Oh SJ, Jung M, Kim YO. Late Bone Metastasis of Histologically Bland Struma Ovarii: The Unpredictability of Its Biologic Behavior. J Pathol Transl Med. 2015; 49(4): 343-345, doi: 10.4132/jptm.2015.04.27, indexed in Pubmed: 26072948.

15. Ukita M, Nakai H, Kotani $Y$, et al. Long-term survival in metastatic malignant struma ovarii treated with oral chemotherapy: A case report. Oncol Lett. 2014; 8(6): 2458-2462, doi: 10.3892/ol.2014.2587, indexed in Pubmed: 25364407.

16. DeSimone CP, Lele SM, Modesitt SC. Malignant struma ovarii: a case report and analysis of cases reported in the literature with focus on survival and I131 therapy. Gynecol Oncol. 2003; 89(3): 543-548, indexed in Pubmed: 12798728

17. Checrallah A, Medlej R, Saadé C, et al. Malignant struma ovarii: an unusual presentation. Thyroid. 2001; 11(9): 889-892, doi: 10.1089/105072501316973163, indexed in Pubmed: 11575860

18. McGill JF, Sturgeon C, Angelos P. Metastatic struma ovarii treated with total thyroidectomy and radioiodine ablation. Endocr Pract. 2009; 15(2): 167-173, doi: 10.4158/EP.15.2.167, indexed in Pubmed: 19289330.

19. Yamashita M, Ishii T, Ohtori S, et al. Metastasis of malignant struma ovarii to the lumbar spine. J Clin Neurosci. 2010; 17(2): 269-272, doi: 10.1016/j.jocn.2009.04.024, indexed in Pubmed: 20036545.

20. Ciccarelli A, Valdes-Socin H, Parma J, et al. Thyrotoxic adenoma followed by atypical hyperthyroidism due to struma ovarii: clinical and genetic studies. Eur J Endocrinol. 2004; 150(4): 431-437, indexed in Pubmed: 15080771.

21. Matsuda K, Maehama T, Kanazawa K. Malignant struma ovarii with thyrotoxicosis. Gynecol Oncol. 2001; 82(3):
575-577, doi: 10.1006/gyno.2001.6315, indexed in Pubmed: 11520159.

22. Rotman-Pikielny P, Reynolds JC, Barker WC, et al. Recombinant human thyrotropin for the diagnosis and treatment of a highly functional metastatic struma ovarii. J Clin Endocrinol Metab. 2000; 85(1): 237-244, doi: 10.1210/jcem.85.1.6261, indexed in Pubmed: 10634393.

23. Hatami M, Breining D, Owers RL, et al. Malignant struma ovarii--a case report and review of the literature. Gynecol Obstet Invest. 2008; 65(2): 104-107, doi: 10.1159/000108654, indexed in Pubmed: 17890867.

24. Makani S, Kim W, Gaba AR. Struma Ovarii with a focus of papillary thyroid cancer: a case report and review of the literature. Gynecol Oncol. 2004; 94(3): 835-839, doi: 10.1016/j. ygyno.2004.06.003, indexed in Pubmed: 15350384.

25. Tang C, Yang L, Wang Ni, et al. High expression of GPER1, EGFR and CXCR1 is associated with lymph node metastasis in papillary thyroid carcinoma. Int J Clin Exp Pathol. 2014; 7(6): 3213-3223, indexed in Pubmed: 25031742.

26. Kaliszewski K, Diakowska D, Strutynska-Karpinska M, et al. Expression of cytokeratin-19 (CK19) in the classical subtype of papillary thyroid carcinoma: the experience of one center in the Silesian region. Folia Histochem Cytobiol. 2016; 54(4): 193-201, doi: 10.5603/FHC.a2016.0025, indexed in Pubmed: 28051274.

27. Sanuvada R, Nandyala R, Chowhan AK, et al. Value of cytokeratin-19, Hector Battifora mesothelial-1 and galectin-3 immunostaining in the diagnosis of thyroid neoplasms. J Lab Physicians. 2018; 10(2): 200-207, doi: 10.4103/JLP, indexed in Pubmed: 29692588.

28. Omary MB, Ku NO, Strnad P, et al. Toward unraveling the complexity of simple epithelial keratins in human disease. J Clin Invest. 2009; 119(7): 1794-1805, doi: 10.1172/JCI37762, indexed in Pubmed: 19587454.

29. Kirfel J, Magin TM, Reichelt J. Keratins: a structural scaffold with emerging functions. Cell Mol Life Sci. 2003; 60(1): 56-71, indexed in Pubmed: 12613658.

30. Chu PG, Weiss LM. Keratin expression in human tissues and neoplasms. Histopathology. 2002; 40(5): 403-439, indexed in Pubmed: 12010363.

31. Kawai T, Yasuchika K, Ishii T, et al. Keratin 19, a Cancer Stem Cell Marker in Human Hepatocellular Carcinoma. Clin Cancer Res. 2015; 21(13): 3081-3091, doi: 10.1158/1078-0432. CCR-14-1936, indexed in Pubmed: 25820415.

32. Liu L, Teng J, Zhang L, et al. The Combination of the Tumor Markers Suggests the Histological Diagnosis of Lung Cancer. Biomed Res Int. 2017; 2017: 2013989, doi: 10.1155/2017/2013989, indexed in Pubmed: 28607926.

33. Wei XQ, Ma Y, Chen Y, et al. Laparoscopic surgery for early cervical squamous cell carcinoma and its effect on the micrometastasis of cancer cells. Medicine (Baltimore). 2018; 97(34): e11921, doi: 10.1097/MD.0000000000011921, indexed in Pubmed: 30142804.

34. Zhang X, Ji C, Gu Z, et al. [Correlation between Serum Cytokeratin 19 Fragment and the Clinicopathological Features and Prognosis of Thymic Epithelial Tumors]. Zhongguo Fei Ai Za Zhi. 2018; 21(7): 519-525, doi: 10.3779/j.issn.10093419.2018.07.03, indexed in Pubmed: 30037371.

35. Bejarano PA, Nikiforov YE, Swenson ES, et al. Thyroid transcription factor-1, thyroglobulin, cytokeratin 7, and cytokeratin 20 in thyroid neoplasms. Appl Immunohistochem Mol Morphol. 2000; 8(3): 189-194, indexed in Pubmed: 10981870.

36. Mills AA, Zheng B, Wang XJ, et al. p63 is a p53 homologue required for limb and epidermal morphogenesis. Nature. 1999; 398(6729): 708-713, doi: 10.1038/19531, indexed in Pubmed: 10227293. 
37. Parsa R, Yang A, McKeon F, et al. Association of p63 with proliferative potential in normal and neoplastic human keratinocytes. J Invest Dermatol. 1999; 113(6): 1099-1105, doi: 10.1046/j.15231747.1999.00780.x, indexed in Pubmed: 10594758.

38. Radu TG, Mogoantă L, Busuioc CJ, et al. Histological and immunohistochemical aspects of papillary thyroid cancer. Rom J Morphol Embryol. 2015; 56(2 Suppl): 789-795, indexed in Pubmed: 26429174.

39. Wegman SJ, Parwani AV, Zynger DL. Cytokeratin 7, inhibin, and p63 in testicular germ cell tumor: superior markers of choriocarcinoma compared to -human chorionic gonadotropin. Hum Pathol. 2019; 84: 254-261, doi: 10.1016/j. humpath.2018.10.007, indexed in Pubmed: 30359635.

40. Marti JL, Clark VE, Harper H, et al. Optimal surgical management of well-differentiated thyroid cancer arising in struma ovarii: a series of 4 patients and a review of 53 reported cases. Thyroid. 2012; 22(4): 400-406, doi: 10.1089/thy.2011.0162, indexed in Pubmed: 22181336.
41. Matysiak-Grzes M, Fischbach J, Gut P, et al. Struma ovarii maligna. Neuro Endocrinol Lett. 2013; 34(2): 97-101, indexed in Pubmed: 23645304.

42. Shrimali RK, Shaikh G, Reed NS. Malignant struma ovarii: the west of Scotland experience and review of literature with focus on postoperative management. J Med Imaging Radiat Oncol. 2012; 56(4): 478-482, doi: 10.1111/j.17549485.2012.02394.x, indexed in Pubmed: 22883659.

43. Zhang X, Axiotis C. Thyroid-type carcinoma of struma ovarii. Arch Pathol Lab Med. 2010; 134(5): 786-791, doi: 10.1043/1543-2165-134.5.786, indexed in Pubmed: 20441513.

44. Leong A, Roche PJR, Paliouras M, et al. Coexistence of malignant struma ovarii and cervical papillary thyroid carcinoma. J Clin Endocrinol Metab. 2013; 98(12): 4599-4605, doi: 10.1210/jc.2013-1782, indexed in Pubmed: 24217901.

45. Barrera JR, Manalo LA, Ang FL. Papillary thyroid-type carcinoma arising from struma ovarii. BMJ Case Rep. 2012; 2012, doi: 10.1136/bcr.03.2012.6145, indexed in Pubmed: 22787184.

Submitted: 4 December, 2018

Accepted after reviews: 25 March, 2019 Available as AoP: 28 March, 2019 
